punch forceps or scissors and snare. A Krause's trocar and cannula is next introduced into the nose and the antrum punctured. The trocar is withdrawn and a blunt pilot introduced, and the inner wall of the antrum broken inward towards the nose. The opening into the antrum can now be seen, and the greater part of the antro-nasal septum can be chipped away. In doing this Luc's ethmoidal forceps cutting iackward and Ostrom's forceps cutting forwards will be found most useful. In enlarging the opening in a forward and upward direction care must be taken not to interfere with the nasal duct, but this is not very likely to happen if ordinary care is taken, for the bone around it is generally hard. The antro-nasal septum is for the most part tormed of a very thin bone, which is easily removed, except below, where a solid ridge is found to separate the two cavities. It is essential that this should be removed as completely as possible, so that any discharge that may collect in the antrum shall run into the nose and be blown out at once. The ridge is most conveniently removed by a special burr, and this instrument is of further value in smoothing off the edges all round the lower part of the opening which has been made. The essential point in the operation is to make the opening as large as possible. The next step is to examine the antral cavity. Where necessary polypi can be curetted away, but, generally speaking, the less the lining mucous membrane is interfered with the more rapid and complete will the recovery be. No packing is required.

Recently the Canfield-Ballinger operation has been advised; it has been pointed out that the removal of the anterior two-thirds of the inferior turbinate decreases the area for warming and moistening the inspired air. In this operation, therefore, the antro-nasal wall is only removed below the attachment of the interior turbinate, but the opening is carried well forward by the removal of part of the nasal process of the superior maxilla, so that the anterior angle of the antrum is well exposed. I have performed this operation a few times, but I have found tbat the opening into the antrum has some tendency to close, and I have never seen any serious trouble follow the removal of the anterior two-thirds of the inferior turbinate. Where operations for the cure of antral suppuration fail, and $I$ have seen many failures, the trouble is almost invariably that too small an opening is made from the antrum to the nose, and I would most strongly urge the complete removal of the bony wall separating the two cavities.

After treatment. - The after-treatment is very simple. On the day following the operation the antrum is washed out with a bent tube passed into it through the nose and a Higginson's syringe. Ordinary nasal lotion is used, and the patient is directed to bend his head forward and breathe through the mouth, and to at once raise his hand if the lotion passes into his pharynx. He very soon learas to pass the antral tube himself, and should syringe out the cavity twice daily. It is essential to impress upon the patient the importance of thoroughly cleansing the antrum, and not until he feels assured that this will be carried out regularly and systematically should the surgeon relax his direct supervision. By the end of three weeks or a month the discharge will have become much less, and it will in most cases be sufficient to thoroughly cleanse the antrum once daily. At the end of three months it will generally be safe to leave off syringing for longer and longer intervals until. with the complete cessation of all discharge, the case may be considered cured.

The ScotTrsh MIDwIVEs' Assocration.-At the lastmeeting of the Council of the Scottish Midwives' Association the question of the relations between the medical practitioner and the registered midwife was discussed. The Association had before it evidence that in Scotland medical practitioners in working-clags practices a vailed themselves of the assistance of the "friendly neighbour" when certified midwife could have been obtained. 'The Council of the Association in these circumstances bave drawn attention to the advantages that would accrue to both mother and infant if the midwives enrolled at the Central Midwives Board were given proper opportunity to carry out their vocation. It is to be hoped that the Association will make public the grounds on which they consider that in Scotland the registered midwife is especially neglected, or the "friendly neighbour" preferred.

\section{OPHTHALMIC PHYSICIANS AND THE ADVANCEMEN'T OF OPHTHALMOLOGY.}

BY RAYNER D. BAT'TEN, M.D., B.S. LoND., SKNIOR SURGEON TO THE WFSTERN OPHTEALMIC HOSPITAL; LATH OPHTHATMIC SURGEON, QUEEN ALEXANDRA MHLTARY HOBPITAL.

THE present paper had its origin when a vacancy recently occurred on the staff of our hospital and I urged that what was really required was an ophthalmic physioian, not an ophthalmic surgeon. My arguments in support of this course were met by the answer that it was "not done" at any other hospital, and, secondly, that there were no men available or educated for such a post. I was then challenged to bring the subject forward for open discussion.

Cases requiring operation or direct surgical treatment form such a small part of the whole of our work that we have to be responsible for a far larger clinic than we can personally attend to if it is to yield sufficient operative material for the study and advancement of that branch of our science. In order to cope with this difficulty large numbers of men are educated, given posts as clinical assistants, and entrusted with a large measure of responsibility. We avail ourselves of their services for years, during which they become able ophtbalmic observers, but are given no training in ophthalmic surgery and no chance of learning to operate, while their training in, and knowledge of, medicine is insufficient to make them ophthalmic physicians.

\section{Progress on Medical Lines.}

Further progress in ophthalmology, as far as one can see, will be mainly on medical lines, and will require men with a physician's training and experience. Progress on surgical lines is limited and can only result from large experience. There may be another and yet another new operation for glaucoma; we can still discuss the best form of cataract extraction; and someone may some day invent a successful operation to cure detachment of the retina or conical cornea, though I doubt if success is likely to come from the surgical side in the treatment of these diseases.

On the medical side, however, there are endless possibilities which we try to tackle with our second-hand scraps of medical knowledge, picked up from such general medical information as may be current, but with no means of studying the problems from a strictly medical standpoint. Of course, we have physicians attached to our ophthalmic hospitals, but their position is that of consulting referees; they do not have patients of their own. To them we refer cases, but the result is not very satisfactory. There is divided responsibility, or more generally the responsibility rests with the surgeon, even in well-marked medical cases, since the eye-symptom is the most obvious factor. This is specially the case in hospital practice. In private practice the responsibility is more often shared, though even there the need of a physician who can grasp the full importance of the ophthalmic problem is acutely felt. In doubtful cases the physician seldom suggests a cause, and, having returned a negative to the question we have asked, the problem ceases to interest him. Where the diagnosis is obvious he shirks treatment, since the eye-symptom is the guiding factor; and where the case is transferred to the physician he is seldom in a position to study it from the ophthalmic point of view. At present our consulting physicians are not appointed for their knowledge of ophthalmology, but for their knowledge in some branch of medicine, generally with a leaning to neurology. We should still require the consulting physician for special cases, but what we require is ophthalmio physicians, who do their own ophthalmic work and have to live, so to speak, with their ophthalmic failures, and to study ophthalmic cases from a physician's point of view and with a physician's experience.

Special Problems for the Physician.

The need of the physician in ophthalmology seems to me so obvirus, that I will not weary you with elaborating the point. but will merely mention some of the most noteworthy instances.

1. The various vasoular conditions; variations in blood pressure, arterio-sclerosis, vascular degeneration, thrombosis,

1 A paper read at a meeting of the Ophthalmological Section $f$ the Royal Society of Medicine on Nov. 5th, 1919.1 
and all the allied conditions associated with renal, hepatic, and cardiac diseases.

2. The diseases of the central nervous system, not only those of more or less obvious central nerve disease-tabes, disseminated sclerosis, cerebral tumours, ocular paralysesbat the many obscure ocular maladies which fall between the surgical and medical sides of ophthalmology; unexplained optic atrophies; headaches of apparently visual origin, but without ocular symptoms as yet within our range of diagnosis.

3. The many toxic or septic conditions giving rise to definite ocular disease, such as irido-cyclitis, in which the present position is most unsatisfactory. At uresent we refer the case to the consulting physician, dentist, pathologist, and all the rest, and get a series of negative reports, and the case comes back to us for empirical treatment; whereas it ought to come back to the physician and live with him until he has found a cause. Our training and means of research are totally inadequate to the scientific investigation of medical cases. Toxic conditions, giving rise to iritis, cataract, retinal changes, in diabetes, albuminuria, syphilis, tubercle, septic and other conditions, and toxæmia from drugs, form a list too long to enumerate.

4. The various nutritional diseases, giving rise to corneal ulcers. These cases I consider peculiarly disgraceful to us, especially the children with recurrent corneal ulcers, which defy local treatment and result in permanent corneal nebulæ.

This classification by no means exhausts the medical side of ophthalmology, but may be sufficient to show how totally inadequate is our education to deal with a very large proportion of our cases. The fact that we are educated as surgeons tends to dull our keenness in and power of investigating the primary causes of certain ophthalmic problems. We are very loth to stray beyond the orbit, and our education is not wide enough to enable us to do so with profit.

Oataract and Glauooma; Myopie Detaohment of the Retina.

Take the case of cataract. Here we know that, in the ultimate result. we can operate to relieve the worst of the symptoms. We look with suspicion on any non-operative system of treating cataract. Most of these systems depend on some local application to the eye, and are not based on any investigation as to the cause. We know too well the fallacies of the results claimed for this or that system of drops "dissolving" cataracts, and we rightly avoid anything that savours so strongly of quackery.

But if we consider the extraordinary variations in the rapidity of progress in some young or middle-aged cases of cataract-how they will start rapidly, and then berome quiescent, or even improve--surely there is sufficient evidence that they are due to some constitutional cause beyond our power of recognition as surgeons. The causation of cataract requires a more subtle investigation than we cin give it.

Take again the glaucomas. I cannot see how any thoughtful man can be satisfied with either the surgical diagnosis or treatment of many of them. A proportion of them are due to anatomical causes, and are relieved or cured by surgical means. But there are a number which defy surgical treatment, and do not exhibit any anatomical cause; yet they progress, their discs show deep cupping without any evidence of increased tension, and we are driven to the supposition that they have increased tension when we are not looking. Some of the acute glancomas, too, show a virulence for which no anatomical explanation is sufficient. While the astual treatment of the state of hypertension must remain in surgical hands, yet there are many questions in causation and treatment which require to be investigated by the physician. It is not to our credit that, glauroma having occurred in one eye, we should be so prolific in theories and so ignorant as to its causation that we are unable to prevent its onset in the other, except by operation. Yet we rarely refer our cases of glaucnma to our physician, probably because we know he is not sufficiently familiar with the condition to be of any real help.

For many years I studied myopias, and convinced myself that they were often due to constitutional causes, taking the view that the sclerotic became soft and yielder to normal pressures. I still think so. But I gave up the subject, because I was not enough of a physician to follow it up.
Here, too, the fact that we can relieve the symptoms by correcting the error of refraction lulls us into thinking that we are treating the disease; which, however, is far from the case.

Our attitude towards detachment of the retina is even more hopeless. Even if we leave out of consideration the detachments in highly myopic eyes, which, according to some, have an anatomical cause, yet still we bave detachments occurring in emmetropes, or in eyes with only a low degree of myopia. A detachment having occurred in one eye, it is more than probable that it will occur in the other. Yet we are incompetent to give any advice, based on knowledge of causation, to prevent such a calamity. Our surgical treatment of these cases is, on the whole, a failure. One per cent. would be a high average of success. Surely we should do well to hand the lot to the physician, and see what he can make of them.

\section{The Education of the Ophthalmologist.}

The education of the ophthalmologist is primarily at fault. We insist in the first place that a man must pass the highest examination in general surgery, which it is true gives us an exceptional to pe of man, but which has practically no bearing on eyes. He wastes years in acquiring the requisite anatomical and surgical knowledge to pass this examination when his time would be better spent in acquiring some knowledge of medicine and his own specialty. It is hy no means unusual to find amongst ophthalmic surgeons men who not only confess to ignorance of the medical side of optsthalmology, but who seem to glory in their detachment from medicine. At present we act as eyes to the physician, but I hold that the physician should see for himself, and he requires a larger experience than can be derived from stadying only speciril cases.

I do not expect much support from ophthalmic surgeons on the medical side of the question, for the surgeon is an extraordinarily self-satisfied man, and generally thinks himself at least the equal of the physician on the medical side of his art, a position justified, perbaps, by the small amount of help at present given by the physician. On the surgical side, however, I hope to carry convictın.

The actual surgical work of the ophthalmologist forms only a small proportion-not more than 1 or 2 per cent.- of the whole mass of work to be done. As at present arranged, any man going in for eyes is supposed to be an "ophthalmic surgeon," and must assume himself, and be assumed to be, capable of doing any ophthalmic operation. Yet what has his training been, and what opportunity has he had of dealing with operative cases? A man may work 10 to 15 years at our leading ophthalmic hospitals and never do a major ophthalmic operation. Yet he is a highly trained ophthalmologist except in operative skill, which he must gain in the scholl of experience-expensive alike to himself and his patients-if he is ever to get on the staff of a ho:pital and become an efficient ophthalmic surgeon. In due course he will have to undertake his first major operatirn. He cannot under the present system decline. He is an ophthalmic surgeon, and he must either undertake the operation or put himself into an inferior rank and pass the case on to another.

The war has brought us many "ophthalmic specialists" who are in no way ophthalmic surgeons. In a case known to me one of them came across a case of cataract. He did not feel himself competent and got another man to " help" him with the operation. Unfortunately, he showed but little wisdom in his choice of a helpmeet. He chose a peculiarly incompetent but confident young man, who had never been trusted to do any but the simplest operations. Between them they operated, with the natural result. I have not heard what happened to the other eye.

\section{Diffuculties of Teaehing Operative Work.}

The question of teaching operative work is a very difficult one. It would obviously be improssible and undesirable to teach operative work to the large number of men passing through our hospitals seeking ophthalmic training. They cannot all become operators. It would be unjust to the patients and to the hospital. Ophthalmic surgery differs from all other surgery in that there is no gradual road to competence. You cannot allow a man to begin an op rati nn. say, for extraction of cataract and, if he gets inte difficulties, step in and set things right. The mischief is 
done, and men have only two eyes. Instructing men in eye operations is a most painful proceeding, even when they have watched and helped you at previous operations. From the introduction of the speculum to its removal there is of ten a series of mistakes and clumsiness, even in such operations as tenotomy, advancement, or excision.

There are many distinguished ophthalmologists who are in no way fitted to be surgeons. They are either innately clumsy, over-confident, or hopelessly nervous and anxious, so that they are quite incapable of doing justice either to themselves or their patients. One of the best ophthalmologists I have known was constitutionally unfit to operate; over-conscientious, he was always unhappy at his operations, and could never do himself jastice; his failures caused him acute distress and sleepless nights. Yet there was no way out for him.

It would, I think, be better for the profession if ophthalmic operations were in fewer hands. Such an arrangement would tend not only to a higher standard of operative skill, but to enterprise and progress in surgical treatment. The surgeon, being no longer required to treat cases essentially medical, would be free to devote himself with a single eye to the advancement and perfection of surgical methods, and would have in addition increased opportunity for their practice. Surgeon and patient alike would benefit. It would then be possible to have private ophthalmic hospitals, accustomed to and equipped for ophthalmic operati ins, instead of being dependent upon nursing-homes, where eye operations are infrequent and the nursing and equipment unsatisfactory. The present position is not fair to the public, and is not to the honour of ophthalmic surgery, nor to its profit. It would be far better for all concerned if we recognised ophthalmic physicians as well as ophthalmic surgeons.

\section{Conclusion.}

My remedies for the present conditions would be :-

1. To improve ophthalmic education.

2. To appoint ophthalmic physicians to our hospitals.

In place of the Fellowship I would substitute an ophthalmic qualification, and it should be possible to qu ilify medically or surgically. The surgical qualification would require evidence of operative skill. Even for an ordinary medical qualification, a man is required to have attended his 20 or 30 cases of midwifery. Is it too much to ask that he should show evidence of some operative skill in ophthalmic surgery before he is admitted as an ophthalmic surgeon?

These changes would take time. But the recognition of ophthalmir physicians and their appointment to hospitals could be effected at once. They should attend out-patients and have beds at their disposal. They should see all classes of cases, but should be relieved of all operative work. The surgeons, on the other hand, would refer their medical cases to the physician. This would provide a larger number of ophthalmic men to deal with the ever-increasing mass of ophthalmic work.

This system would, I beliere, be of advantage both to the physicians and surgeons, both in hospital and private work, and would redound to the credit and advancement of ophthalmology.

A Medical APPONNTMENT AT OMAGH.-Extraordinary interest has been manifested by the Omagh Guardians in the election of a medical officer for one of the dispensary districts. On Nov. 22nd, out of a total board of 84 there were 83 members in attendance, to decide the fate of two candidates-Dr. B. Lagan and Mr. J A. Cunningham -who at a previous election had achieved a tie, each receiving 40 votes, and so rowdy was the meeting on that occasion that the election was adjourned. On the second occasion Dr. Lagan received 42 votes and Mr. Cunningham 41 ; but before the election a letter was received from a solicitor, giving notice that three members of the board were disqualifed from voting, and claiming that their votes should not be accepted. According to report they had voted for the candidate who was elected, in which case the report amounts to a suggestion that the election may still be challenged; but, to quote a local paper: "Beyond a few angry words nothing untoward occurred, and the services of a body of policemen who were in the vicinity of the workhouse in the event of disturbances were not called for."
THE VALUE OF THE

COMPLEMENT-FIXATION TEST IN GONOCOCCAL INFECTIONS.

By H. B. F. DIXON, M.C., M.B., B.CH. DUE., AND

A. H. PRIESTley, M.B., Ch.B. Vict., CA P'TAINS, R.A.M,C.

THE syphilologist has a great advantage over the specialist in gonorrbcea, both in ihe diagnosis of obscure cases and in treatment. In diagnosis he has the Wassermann test, which is not only of the greatest service in elucidating obscure and latent cases, but also serves to show whether treatment has been effective, and when repeated at intervals whether the apparent cure has been maintained. In treatment he has drugs having a specific action on the spironema. The clinician who treats gonorrhoea has no such aids, though gonorrhoea is quite as serious a diseasa as syphilis, and there are without doubt far more cases of unsuspected gonorrhœa than of latent syphilis.

In dealing systematically with a large number of cases of gonorrhœa we have been struck by two difficulties which hardly occur in syphilis. In the first place there is the difficulty of establishing a diagnosis in the absence of gonococci in the urethral discharge, or even in the presence of Gram-negative cocci where there is no time or opportunity for culture. In the second place there is the difficulty of establishing a standard of cure. The most stringent clinical standard cannot approach in accuracy a series of serum tests. The utmost the clinician can do in gonorrhoea is to attempt in an apparently cured case by means of provocative injections of vaccine, silver-nitrate injections, instrumentation, prostatic massage, \&c., to cause the reappearance of pus cells and gonococci. If no pus and no gonococci appear the patient is assumed to be cured; nevertheless, relapses do occur.

It must be remembered that the complement-fixation test in gonorrboea is not comparable, except to some extent in technique, with the Wassermann test. This is a point often overlooked, yet of primary importance in the interpretation of results. The Wassermann is a test of the damage done to the tissues by the spironema. If this has not gone too far complete destruction of the organism causes cessation of damage, and a negative reaction follows. The serum test in gonorrhœe is a true antibody test, and is therefore not a test of damage but of resistance. It is thus comparable to Nogucbi's true spironema antigen test, which becomes positive as the patient improves. The wide range of the Wassermann test also renders it more useful than the gono. coccus test can be. In syphilis it is not uncommon to find sera which, with heart extract (and especially cholesterinised extract), will fix 20 or more doses of complement. In gonorrhoea it is rare to find more than 6 or 7 doses fixed; the highest positive we have met with, excluding cases where large doses of vaccine have been given, fixed 10 doses.

Complement-fixation Test in the Determination of Cure.

We began this study in the hope that in the complementfixation test we might find help in both the diffinulties alluded to-namely, in diagnosis and, more particularly, in determining the grave question as to whether a patient can really be considered cured, without merely turning him out in the hope that he is cured. We think that we have evidence to prove the definite utility of the test in diagnosis. As regards a test of cure we at present hold open minds, but we have formed three very definite impressions, viz. :-

1. That no person suffering from gonorrhoea can be regarded as doing well and on the way to cure until he has shown a strong positive reaction, independent of an artificial reaction the result of large doses of vaccine. (What we call a strong positive reaction will appear subsequently.) The majority of our cases have either had no vaccine or only small doses of stock polyvalent gonococcus vaccine-not more than 600 millions. Such doses do not, in our experience, produce a strong positive, so that such a reaction in these cases may be considered to be an indication of a genuine imnunising response on the part of the patient.

2. Detoxicated vaccines, on account of the much higher dosage employed, entirely obscure the immunising response. 